\title{
Editorial
}

\section{Remote Sensing of Soil}

\author{
Mehrez Zribi, ${ }^{1}$ Nicolas Baghdadi, ${ }^{2}$ and Michel Nolin ${ }^{3}$ \\ ${ }^{1}$ CESBIO (CNRS/IRD/UPS/CNES), 18 Avenue Edouard Belin, 31401 Toulouse, Cedex 9, France \\ ${ }^{2}$ TETIS/CEMAGREF, 500 Rue Jean-François Breton, 34093 Montpellier, Cedex 5, France \\ ${ }^{3}$ Agriculture et Agroalimentaire Canada, 979 Avenue de Bourgogne, Québec, QC, Canada G1W 2L4 \\ Correspondence should be addressed to Mehrez Zribi, mehrez.zribi@ird.fr
}

Received 21 November 2011; Accepted 21 November 2011

Copyright (C) 2011 Mehrez Zribi et al. This is an open access article distributed under the Creative Commons Attribution License, which permits unrestricted use, distribution, and reproduction in any medium, provided the original work is properly cited.

Over the past few decades, the Earth's surface has witnessed major changes in land use. These changes are likely to continue, driven by demographic pressure or by climate change. In this context, monitoring tools are needed for maintaining a sustainable ecological status, improving soil conservation and water resource management. Floods, excess runoff, soil erosion, and related contamination and disequilibrium of the water and carbon cycles are, among others, key issues that are controlled and influenced by soil surface characteristics. The implementation of sustainable agricultural, hydrological, and environmental management requires an improved understanding of the soil, at increasingly finer scales. Conventional soil sampling and laboratory analyses cannot efficiently provide this information, because they are slow, expensive, and could not retrieve all temporal and spatial variabilities.

In this context, remote sensing has shown a high potential in soil characteristics retrieving in the last three decades. Different methodologies have been proposed for the estimation of soil parameters, based on different remote sensing sensors and techniques (passive and active). For passive remote sensing, we can consider four principal types of sensors:

(i) optical remote sensing with limited number of bands (e.g., SPOT, ASTER, LANDSAT..., etc.) particularly adapted for vegetation cover description, land use analysis,

(ii) optical remote sensing based on hyperspectral sensors, particularly adapted for soil texture description,

(iii) optical remote sensing with thermal infrared band, adapted for soil temperature estimation, (iv) passive microwave remote sensing adapted to soil moisture and vegetation estimation.

For active remote sensing, different studies have shown a considerable potential for the characterization of different soil parameters: moisture, roughness, and texture. Active remote sensing is particularly based on two types of sensors: synthetic aperture radar (SAR) with high spatial resolution adapted to local and regional studies and scatterometer sensor more adapted to global estimations of soil parameters.

Three types of methodologies are generally used for soil parameters estimation: empirical models based only on satellite and ground databases, semiempirical models based on a mixture between physical modelling and real data, and finally physical models based only on the description of radiative transfert physics to analyze relationship between remote sensing signals and soil parameters.

These remote sensing studies concern particularly four soil parameters (moisture, roughness, temperature, and texture).

(i) Soil moisture is a key parameter, influencing the manner in which rainwater is shared between the phenomena of evapotranspiration, infiltration, and runoff.

(ii) Soil surface roughness is involved in the separation of water flow into infiltration and runoff. Moreover, monitoring the evolution of surface roughness is a way to estimate erosion risk particularly in agricultural areas.

(iii) Soil texture is one of the most important soil properties influencing most physical, chemical, and biological soil processes. Hence, it is a key property for soil management. 
(iv) Soil temperature is a key parameter in the description of evapotranspiration and surface-atmosphere interface processes.

Based on this high potential of remote sensing to retrieve surface parameters, a high number of sensors have been launched in the last years to improve different methodologies proposed to retrieve operationally surface parameters.

In this context, our principal objective of this special issue is to present different studies illustrating quantitative analyses based on these different new sensors.

Pasolli et al. propose a technique for estimating soil moisture based on the support vector regression algorithm and the integration of ancillary data, using active remote sensing (RADARSAT 2 SAR data).

Liu et al. investigated the impact of soil moisture on gross primary production (GPP), chlorophyll content, and canopy water content represented by remotely sensed vegetation indices (VIs) in an open grassland and an oak savanna in California.

Using SAR (RADARSAT-1) remote sensing, Khaldoune et al. provide a classification of frozen/unfrozen soils in the entire Bras d'Henri River watershed near Quebec City (Quebec, Canada). It was developed to produce frozen soil maps under snow cover.

Browning and Duniway present a method to map soils with Landsat ETM+ imagery and high-resolution $(5 \mathrm{~m})$ terrain (IFSAR) data. They then characterize soil classes mapped using this semiautomated technique. The method distinguished spectrally distinct soil classes that differed in subsurface rather than surface properties.

Baptista et al. study tested the feasibility of applying AVIRIS sensor (Airborne Visible/InfraRed Imaging Spectrometer) for mapping and quantifying mineralogical components of three Brazilian soils. They showed to be possible mapping and quantifying the weathering degree of the studied soils.

Hively et al. propose the use of airborne hyperspectral imagery to map tilled agricultural fields properties. Soil hyperspectral reflectance imagery was obtained using an airborne imaging spectrometer $(400-2450 \mathrm{~nm}, \sim 10 \mathrm{~nm}$ resolution, $2.5 \mathrm{~m}$ spatial resolution). The resulting raster maps showed variation associated with topographic factors, indicating the effect of soil redistribution and moisture regime on in-field spatial variability.

Finally, Oguro et al. discuss brightness temperature (BT) measurements and statistics over Taklimakan Desert. They are retrieved from the data of Landsat-7/ETM+ band 6 and Terra/MODIS band 31 and 32 .

In spite the limited number of scientific papers and the difficulty to cover all techniques of remote sensing of soil, we hope that the readers will find this as a useful source of information.

\section{Acknowledgments}

We would like to thank the reviewers who helped us in reviewing the articles and timely recommendations. We also would like to thank the staff of the Editorial Section of
Applied and Environmental Soil Science (AESS), who had faith in us and cooperated at all stages of compilation.

Mehrez Zribi

Nicolas Baghdadi

Michel Nolin 

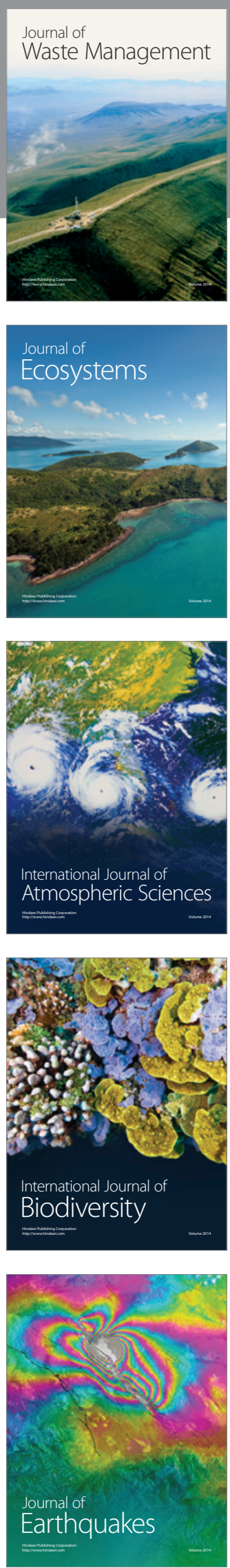
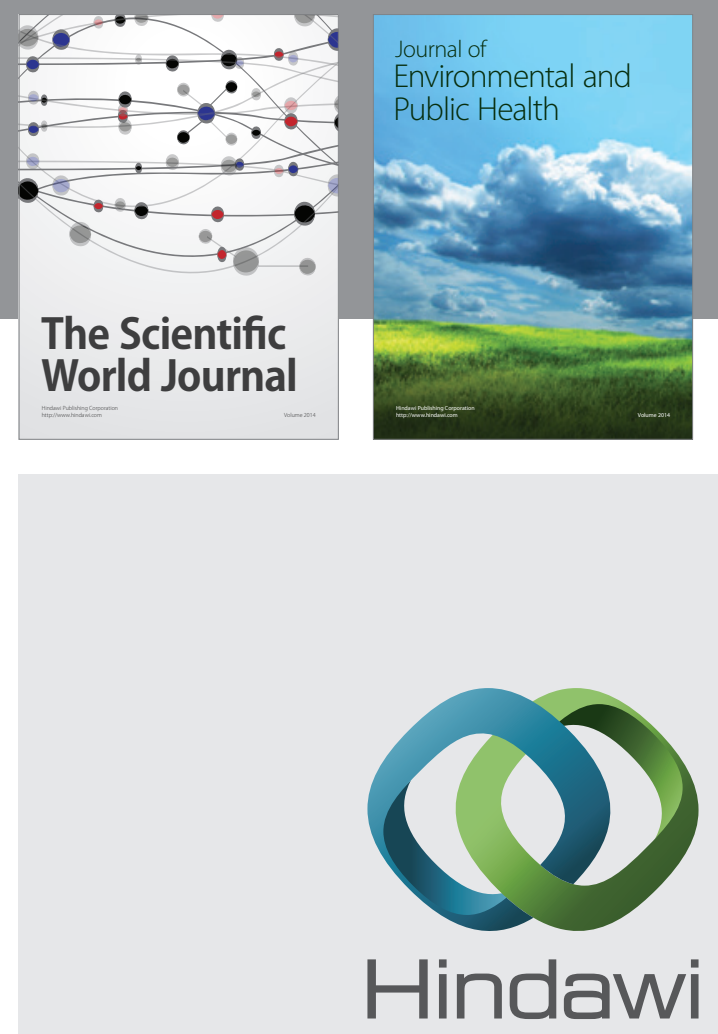

Submit your manuscripts at

http://www.hindawi.com
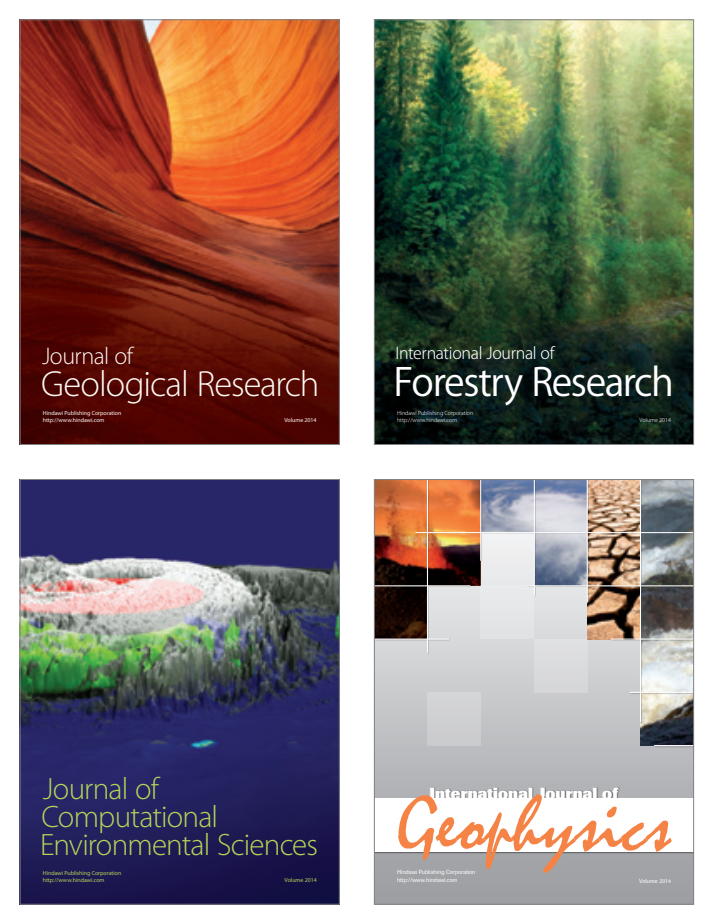
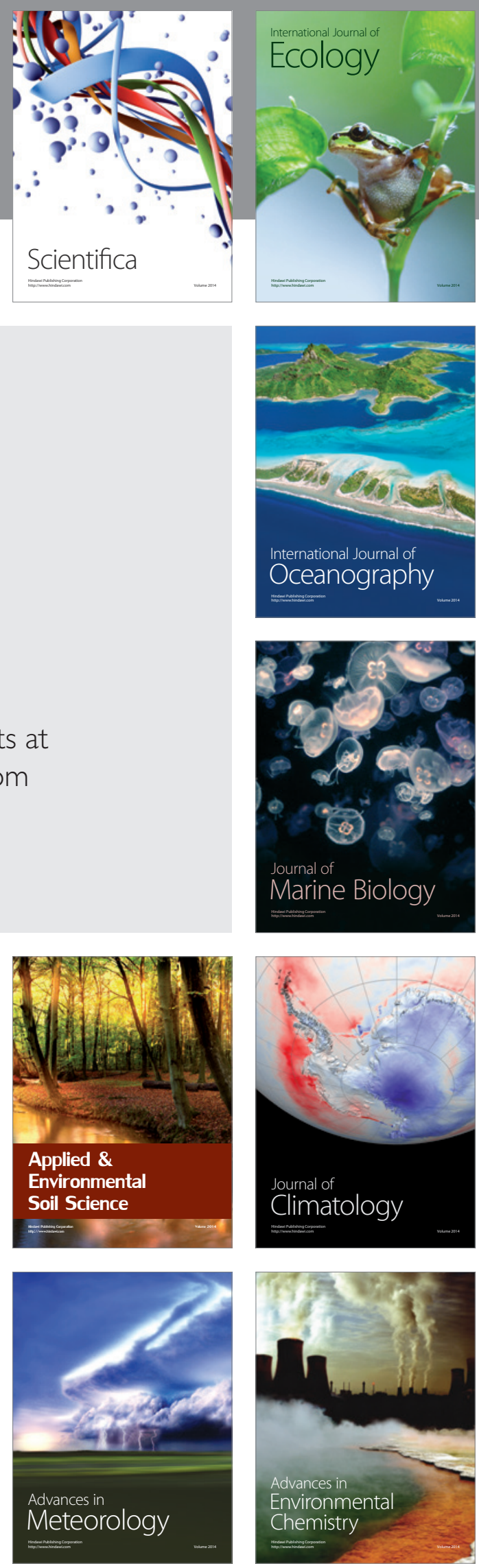